\title{
MONITORIZADO DE LA CONDICIÓN DEL ACEITE DE MOTORES DE AUTOMOCIÓN MEDIANTE UN KIT DE PRUEBAS RÁPIDAS
}

\section{AUTOMOTIVE ENGINE OIL CONDITION MONITORING THROUGH A RAPID TEST KIT}

\section{MSc. Juan Carlos Delgado Sanabria, PhD. Simón de Jesús Fygueroa Salgado PhD. Rafael Bolívar León}

\author{
Universidad de Pamplona, Facultad de Ingenierías y Arquitectura. \\ Programa de Ingeniería Mecánica. \\ Km 1. Vía Bucaramanga. Ciudad Universitaria, Pamplona, Norte de Santander, Colombia. \\ Teléfono: $(57+7) 5685303$ Ext. 164. \\ E-mail: \{jucadesa, sjfigueroa, rbolivarl\}@ unipamplona.edu.co.
}

\begin{abstract}
Resumen: El objetivo del trabajo es evaluar la condición y contaminación del lubricante de un grupo de motores de automoción mediante el monitorizado de algunas de sus propiedades. Se utiliza un kit de pruebas rápidas desarrollado por los autores con el cual se detectan algunos síntomas del motor que varían con el tiempo y a partir de éstos, se establecen relaciones que se pueden utilizar para tomar decisiones respecto a su mantenimiento. El estudio efectuado consistió en extraer periódicamente muestras de aceite a un grupo de motores de automóvil y realizarles los ensayos que permite el kit de pruebas rápidas: mancha de aceite, crepitación, viscosidad y detección de partículas magnéticas. Para calificar los resultados de la mancha de aceite se emplea como parámetro de caracterización el índice de dispersión que de acuerdo con los resultados puede usarse como criterio para determinar el periodo de cambio de aceite. De la prueba de detección de partículas ferromagnéticas se concluye que en los motores diésel se observan a simple vista partículas de desgaste, mientras que en los motores de gasolina es necesario recurrir al uso de un microscopio para percibirlas.
\end{abstract}

Palabras clave: monitorizado, dispersión, viscosidad, partículas ferromagnéticas, crepitación.

\begin{abstract}
The objective of the work is to evaluate the lubricant condition of a group of automotive engines by monitoring some of its properties and its contamination. A rapid test kit developed by the authors is used with which some engine symptoms that vary with time are detected and from these, relationships are established that can be used to make decisions regarding its maintenance. A rapid test kit developed by the authors is used to detect some engine symptoms that vary with time and from these, relationships are established that can be used to make decisions regarding its maintenance. The study consisted of periodically extracting oil samples from a group of automobile engines and carrying out the tests that the rapid test kit allows: oil blotter, crepitation, viscosity and magnetic particle detection. To qualify the oil blotter results, the dispersion index is used as the characterization parameter, which according to the results can be used as a criterion to determine the oil change period. From the ferromagnetic particle detection test it can be concluded that in diesel engines wear particles are visible to the naked eye, while in gasoline engines it is necessary to use a microscope to perceive them
\end{abstract}




\section{INTRODUCCIÓN}

En el mantenimiento predictivo de motores, una de las herramientas más importantes para predecir fallas la constituye el análisis del aceite, que suministra valiosa información utilizable como sintomatología para diagnosticar tanto el desgaste interno del motor como el estado de su lubricante (Fygueroa et al., 2009). El análisis y monitorizado de los síntomas del aceite, brinda suficiente orientación sobre el estado en que se encuentra el motor con lo cual, se pueden prevenir fallas y paradas no planificadas. En el país esta técnica se encuentra poco desarrollada, debido a los pocos laboratorios que prestan sus servicios para este fin y a los altos costos fijos que representan para las empresas (Fygueroa, 2005). Por este motivo los autores, en el Programa de Ingeniería Mecánica de la Universidad de Pamplona, han desarrollado un equipo de pruebas rápidas (EPR) para ser utilizado en la determinación fácil y expedita de algunos síntomas característicos del estado del lubricante y el motor (Delgado et al., 2016).

\section{MATERIALES Y MÉTODOS}

Se emplea una estrategia de trabajo de campo, ya que las pruebas de seguimiento mediante la medición de los diferentes síntomas del aceite, se realizan en el propio ambiente donde se encuentran los automóviles seleccionados que serán objeto de análisis.

\subsection{Equipo de pruebas rápidas}

El equipo de pruebas rápidas consta de una placa de calentamiento, un viscosímetro de caída de bolas y un dispositivo de detección de partículas ferrosas. Con estos aparatos se pueden determinar algunos síntomas característicos de la condición en que se encuentran el lubricante y el motor. El ensayo de crepitación permite evidenciar la presencia de agua en el aceite (Widman, 2005), la mancha de aceite los contaminantes presentes producidos por la combustión y su detergencia/dispersividad (Abellaneda, 2008), el ensayo de viscosidad proporciona información sobre el valor de esta propiedad del aceite y la detección de partículas facilita el análisis de las partículas de desgate presentes en el aceite y determinar su origen (Clymer et al., 1992).

\subsection{Población de estudio}

Para la realización del presente trabajo, se seleccionaron 5 vehículos de servicio público de la ciudad de Pamplona cuyas características se presentan en la Tabla 1, 3 con motor diésel y 2 con motor de gasolina. Cada vehículo se designa con la letra $\mathrm{N}$ seguida de un dígito del 1 al 5 .

Tabla 1: Características de los vehículos seleccionados

\begin{tabular}{|c|c|c|c|c|}
\hline Cod. & Marca & Motor & Tipo & Aceite \\
\hline N1 & Chevrolet & $\begin{array}{l}\text { 4HK1- } \\
\text { TC }\end{array}$ & Diésel & $\begin{array}{l}\text { Mobil } \\
\text { Delbac } \\
15 \text { W44 }\end{array}$ \\
\hline N2 & Chevrolet & $\begin{array}{l}\text { 4JJ1- } \\
\text { TCC }\end{array}$ & Diésel & $\begin{array}{l}\text { Ursa } \\
\mathrm{Td} \\
15 \mathrm{~W} 40\end{array}$ \\
\hline N3 & Chevrolet & $\begin{array}{l}\text { 4JJ1- } \\
\text { TCC }\end{array}$ & Diésel & $\begin{array}{l}\text { Ursa } \\
\text { Td } \\
15 \mathrm{~W} 40\end{array}$ \\
\hline N4 & Kia & $\begin{array}{l}\text { DOHC- } \\
16 \mathrm{~V}\end{array}$ & Gasolina & $\begin{array}{l}\text { Ursa } \\
\text { Td } \\
15 \mathrm{~W} 40\end{array}$ \\
\hline N5 & Chevrolet & $\begin{array}{l}\text { DOHC- } \\
16 \mathrm{~V}\end{array}$ & Gasolina & $\begin{array}{l}\text { Super } \\
1000 \\
25 \mathrm{~W} 60\end{array}$ \\
\hline
\end{tabular}

\subsection{Cronograma de toma de muestras}

En la Tabla 2 se presenta el recorrido por ruta, día, semana y mes y el tipo de motorización: diésel o gasolina de los vehículos seleccionados.

\section{Tabla 2. Recorrido de los vehículos}

\begin{tabular}{llllll}
\hline Tipo & Rutas/d. & $\mathbf{k m} / \mathbf{r u t a}$ & $\mathbf{k m} / \mathbf{d}$. & $\mathbf{k m} / \mathbf{s e m}$. & $\mathbf{k m} / \mathbf{m e s}$ \\
\hline Diesel & $14-16$ & 15 & $210-240$ & $1470-1680$ & $5880-6720$ \\
Gasolina & $1-2$ & 130 & $130-260$ & $910-1820$ & $3640-7280$ \\
\hline
\end{tabular}

Analizando el contenido de la Tabla 2, se observa que los kilómetros recorridos por mes son aproximadamente los recomendados por los fabricantes para realizar el cambio de lubricante. Por este motivo se establece un cronograma de toma de muestras semanal; lapso durante el cual se estima que cumplirán una cuarta parte del recorrido hasta el cambio de aceite. A las muestras tomadas durante sucesivos cambios de aceite se les asigna una letra $\mathrm{T}$ seguida de un número consecutivo y a las muestras semanales tomadas entre cambios, una letra $\mathrm{S}$ seguida de un número consecutivo.

\subsection{Procedimiento de toma de muestras}


Para extraer las muestras de aceite se utiliza una jeringa de succión, Figura 1(a), que permite extraer el aceite del cárter o depósito principal aún con el motor en funcionamiento. Para que la muestra sea representativa y contenga información válida se la debe tomar a través del tubo de medición del nivel de aceite, a la temperatura de operación con el motor en funcionamiento o hasta media hora después de su parada; los envases que la contienen, Figura 1(b), deben hallarse limpios y libres de humedad y se debe extraer un mínimo de $10 \mathrm{ml}$ de aceite que es la cantidad recomendada para realizar las pruebas (Bilbao, 2005).

\subsection{Procedimiento de ensayo con el equipo de pruebas rápidas.}

A cada uno de los vehículos en estudio se le realizaron las pruebas que permite efectuar el EPR según lo describen Delgado et al (2016). El ensayo de la mancha de aceite se llevó a cabo durante tres meses tomando cuatro muestras por semana; las manchas obtenidas se fotografiaron digitalmente en condiciones de luz y distancia focal controladas y se caracterizaron dimensionalmente acotándolas con el software CorelDraw X3. Todas estas muestras se sometieron adicionalmente a la prueba de crepitación.

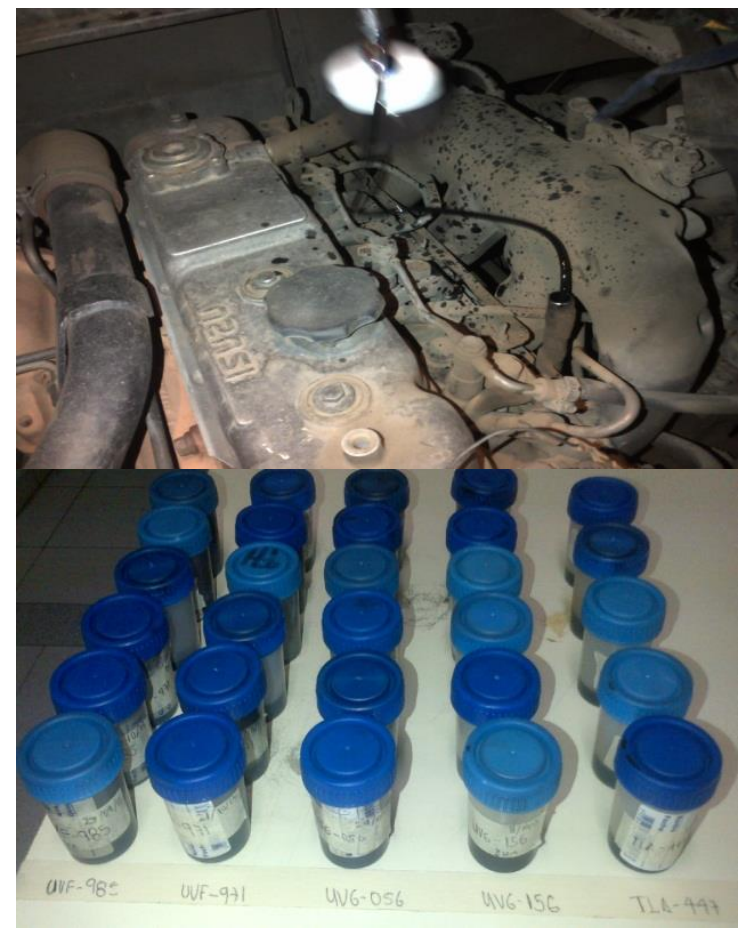

Figura 1. (a) toma de la muestra; (b) muestras de un motor.
A las muestras tomadas cada cambio de aceite se les realizó la prueba de detección de partículas ferromagnéticas, se llevó un registro escrito de lo observado a simple vista en la placa y se efectuó un seguimiento fotográfico para tener un registro visual de los resultados. Al aceite limpio y a las muestras tomadas cada cambio de aceite se les efectuó la prueba de viscosidad (Sam et al, 2006).

\subsection{Caracterización de las manchas de aceite}

La mancha de aceite que tiene la apariencia que se muestra en la Figura 2 presenta dos zonas concéntricas de forma elíptica: la interna de difusión de partículas carbonosas y la externa de difusión del aceite. (Fygueroa et al, 2006) La imagen fotográfica de la mancha se caracteriza dimensionalmente acotando con el software CorelDraw X3 los diámetros mayor y menor de las dos zonas de la mancha, como se observa en la figura.

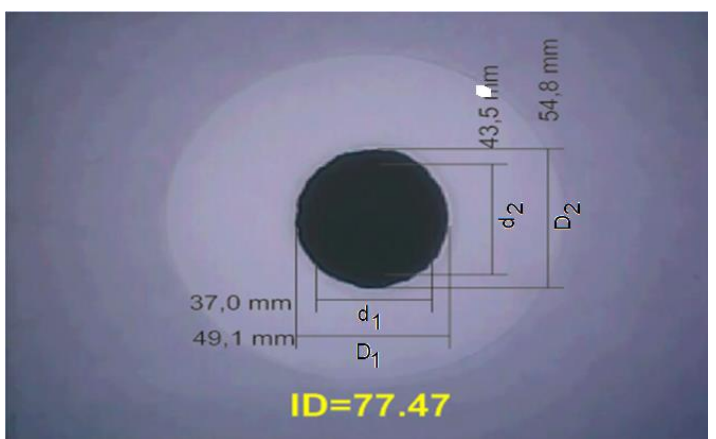

Figura 2. Mancha de aceite del motor N1 acotada

La caracterización de la detergencia/dispersividad de la mancha de aceite se hace mediante el índice de dispersión (ID) que se define mediante la expresión:

$I D=\frac{d}{D}$

donde $d=\frac{d_{1}+d_{2}}{2}$ y $D=\frac{D_{1}+D_{2}}{2}$

son respectivamente los diámetros medios de difusión de las partículas carbonosas y de difusión del aceite.

\subsection{Equipos y software utilizados para el análisis.}

Los resultados de los ensayos se obtuvieron con la ayuda de los siguientes programas y equipos: Microsoft Excel, CorelDraw X3, microscopio Bosh y viscosímetro Brielfield ED. 


\section{RESULTADOS}

Después del monitorizado al lubricante de los motores seleccionados durante tres meses, con el EPR, se obtuvieron una serie de resultados por prueba, que se exponen y analizan a continuación y que sirven como base para conocer el estado de los motores.

\subsection{De la prueba de la mancha de aceite}

Las imágenes de las manchas de aceite de cada vehículo se caracterizan dimensionalmente y se grafican los ID vs el tiempo de muestreo como se muestra en la Figura 3 para una de las muestras.

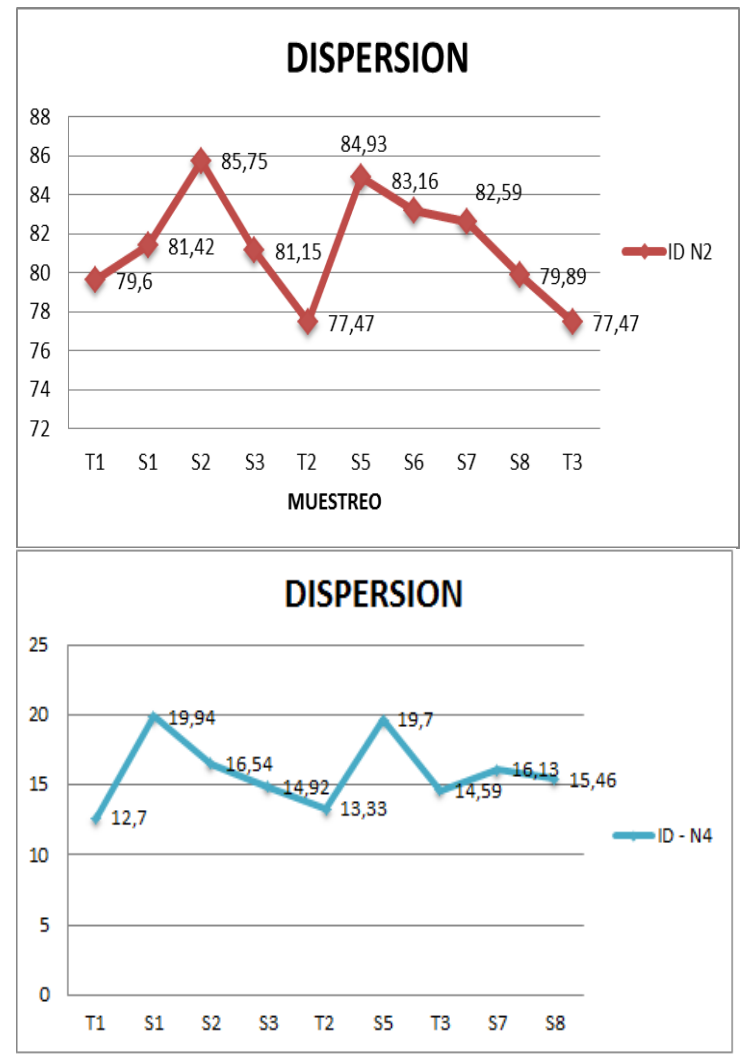

Figura 3. (a) Muestreo vs índice de dispersión motor diésel (N2), (b) Muestreo vs índice de dispersión motor de gasolina (N4).

Analizando la Figura 3(a) se observa que el cambio de aceite se está realizando en el intervalo de kilometraje correcto, ya que los picos más bajos de ésta corresponden a la muestra de cada cambio. Vale destacar que la dispersión disminuye paulatinamente cada semana, comportamiento normal que pone de manifiesto la pérdida de las características dispersantes del lubricante. Para los equipos con motor de gasolina se observa la misma tendencia en la Figura 3(b)

\subsection{De la prueba de crepitación}

Los resultados de la prueba de crepitación para los vehículos con motor diesel revelan que en las muestras de las dos primeras semanas no hay presencia de agua, pero a partir de la tercera semana hasta el cambio aparecen pequeñas burbujas de entre 0.2 y $0.5 \mathrm{~mm}$ como se observa en la Figura 4(a), que no crecen ni crepitan por lo que se infiere que es una humedad normal en el aceite, para estos equipos en la zona que trabajan. Los resultados de la prueba realizada a los motores de gasolina, muestran desde la primera semana después de realizado el cambio de aceite, burbujas de $0.2 \mathrm{~mm}$ como se observa en la Figura 4(b). Puesto que esta humedad no aumenta significativamente ni perjudica las condiciones del aceite ni del equipo, se deduce que es normal.
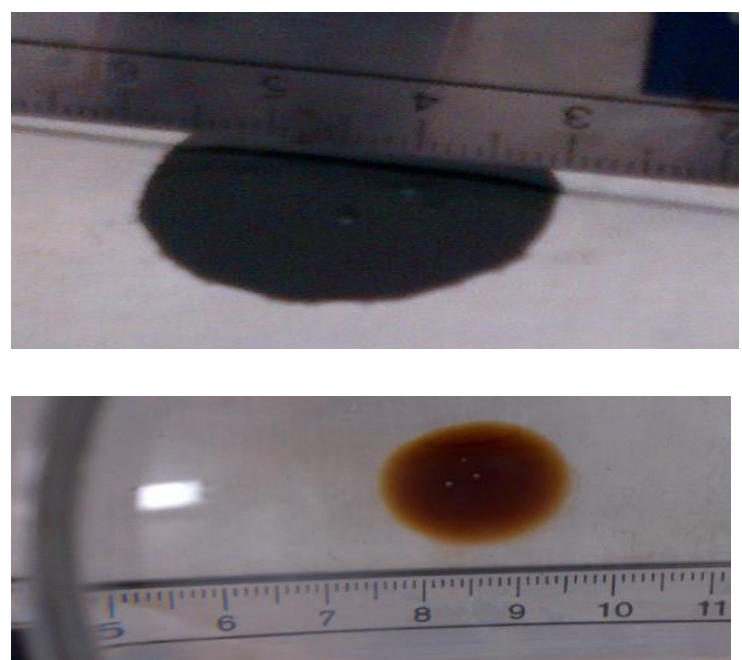

Figura 4. (a) Visualización de la prueba de crepitación para motor diesel, (b) Visualización de la prueba de crepitación para motor de gasolina.

\subsection{De la prueba de partículas ferromagnéticas}

La prueba de partículas ferromagnéticas se realiza a cada una de las muestras del cambio de aceite. Los resultados obtenidos, observables a simple vista o con ayuda de lupa, permiten detectar la presencia de partículas de hierro en el lubricante, ya que los metales y fibras se observan como puntos tal como se muestra en la Figura 5.

Del mismo modo con la ayuda del microscopio Bosh utilizando aumentos de $20 \mathrm{X}$ a $50 \mathrm{X}$, se 
observan las partículas de hierro. Las imágenes obtenidas mediante esta prueba se aparecen en la Figura 6. La primera muestra del equipo N1 con el microscopio pone de manifiesto que hay algunas hojuelas, partículas de corte y pequeñas cadenas de metal como se observa en dicha figura.

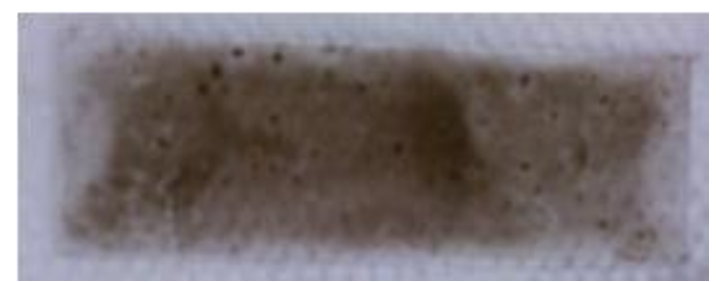

Figura 5. Placa obtenida con el equipo de partículas ferromagnéticas

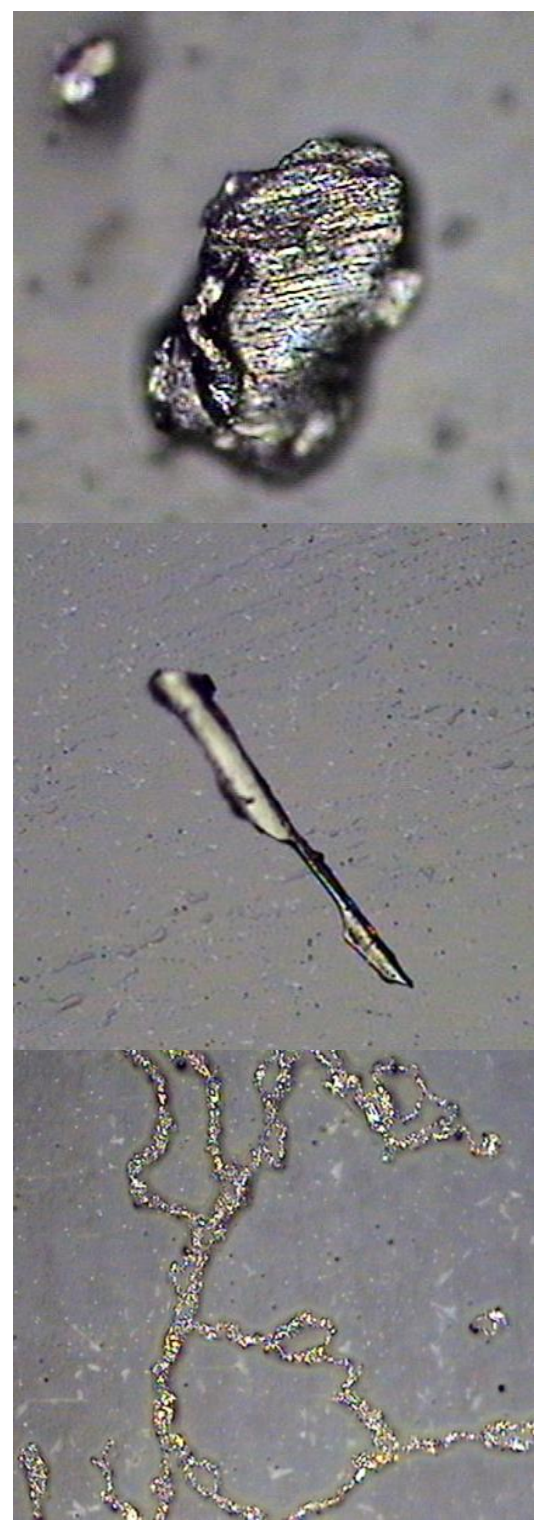

Figura 6. Partículas ferromagnéticas en el equipo N1 (mиеstra T1).
Las muestras del equipo N2 presentan partículas similares a las del equipo N1 tales como cadenas metálicas, fibras, materiales de corte y hojuelas

como se muestra en la Figura 7.

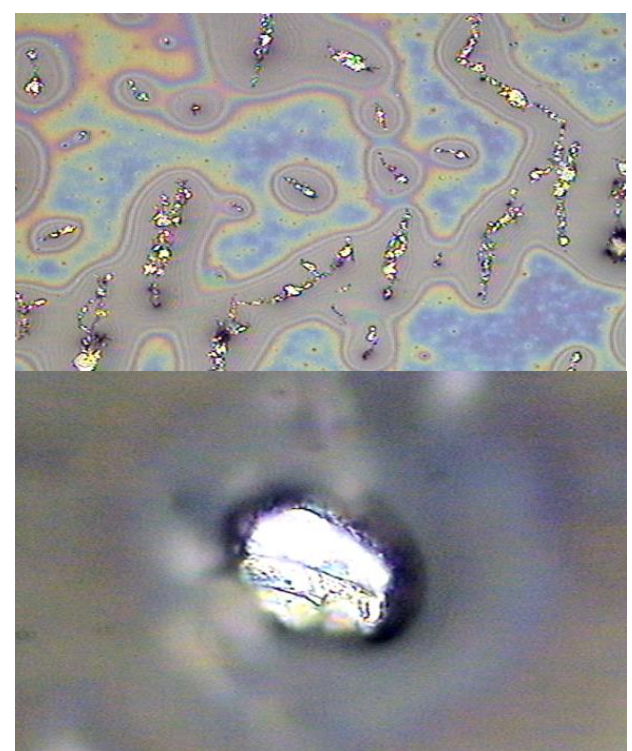

Figura 7. Partículas ferromagnéticas en el equipo N2 (muestra T1).

Los resultados de las pruebas realizadas al vehículo N3 por el contrario, muestran baja cantidad de hojuelas, cadenas metálicas y fibras como se puede apreciar en la Figura 8. Para los vehículos N4 y N5 (con motor de gasolina) utilizando el mismo procedimiento con el que se probaron los equipos diesel no se obtuvo ningún tipo de partículas.

\subsection{Del ensayo de viscosidad}

Este ensayo se realiza a cada una de las muestras del cambio de aceite para comparar su viscosidad con la del aceite limpio y determinar si aumentó o disminuyó.

Como se observa en la Tabla 3 en el equipo N1 la viscosidad cinemática aumenta un $8,5 \%$; aumento que se mantiene de cambio a cambio.

Como se deduce de las Tablas 4 y 5 en los equipos N2 y N3 la viscosidad cinemática aumenta un 8,1 $\%$ y este aumento se mantiene de cambio a cambio En estos equipos se obtienen los mismos resultados debido a que poseen las mismas características de modelo, año, recorrido de trabajo y utilizan aceite de la misma marca y tipo. 

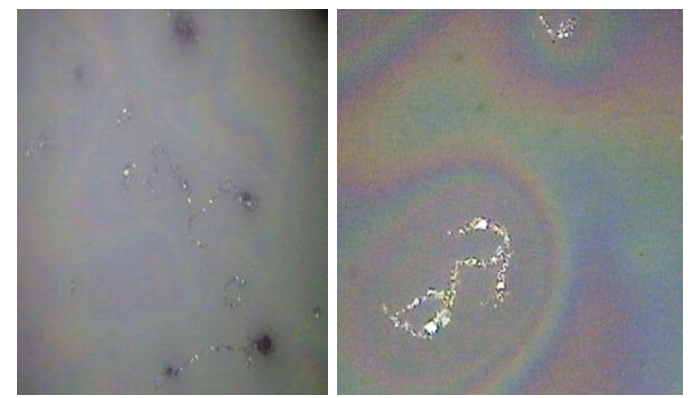

Figura 8. Partículas ferromagnéticas en el equipo N3 (muestra T1).

Tabla 3. Resultados de viscosidad del equipo N1 obtenidos con el viscosímetro de caída de bola.

\begin{tabular}{ccccc}
\hline Ensayo & $\begin{array}{c}\text { Temperatura } \\
{\left[{ }^{\circ} \mathbf{C}\right]}\end{array}$ & $\begin{array}{c}\text { Distancia } \\
{[\mathbf{m m}]}\end{array}$ & $\begin{array}{c}\text { Tiempo } \\
{[\mathbf{s}]}\end{array}$ & $\begin{array}{c}\text { Visc. } \\
\text { Cinemática } \\
{[\mathbf{c S t}]}\end{array}$ \\
\hline Limpio & 22,7 & 17,7 & 0,39 & 255,10 \\
$\mathbf{1}$ & 22,7 & 17,7 & 0,40 & 276,67 \\
$\mathbf{2}$ & 22,7 & 17,7 & 0,40 & 276,67 \\
$\mathbf{3}$ & 22,7 & 17,7 & 0,40 & 276,67 \\
\hline
\end{tabular}

Tabla 4. Resultados de viscosidad del equipo N2 obtenidos con el viscosímetro de caída de bola.

\begin{tabular}{cccccc}
\hline $\begin{array}{l}\text { Ensa } \\
\text { yo }\end{array}$ & $\begin{array}{c}\text { Temperat } \\
\text { ura } \\
{\left[{ }^{\circ} \mathbf{C}\right]}\end{array}$ & $\begin{array}{c}\text { Distan } \\
\text { cia } \\
{[\mathbf{m m}]}\end{array}$ & $\begin{array}{c}\text { Tiem } \\
\text { po } \\
{[\mathbf{s}]}\end{array}$ & $\begin{array}{c}\text { Visc. } \\
\text { Dinám } \\
\text { ica } \\
{[\text { [Pa.s] }}\end{array}$ & $\begin{array}{l}\text { Visc. } \\
\text { Cinemát } \\
\text { ica } \\
{[\mathbf{c S t}]}\end{array}$ \\
\hline $\begin{array}{c}\text { Limp } \\
\text { io }\end{array}$ & 23 & 18,2 & $0,40 \mathrm{~s}$ & 23,9 & 262,35 \\
$\mathbf{1}$ & 23 & 18,2 & $0,41 \mathrm{~s}$ & 24,9 & 283,58 \\
$\mathbf{2}$ & 23 & 18,2 & $0,41 \mathrm{~s}$ & 24,9 & 283,58 \\
$\mathbf{3}$ & 23 & 18,2 & $0,41 \mathrm{~s}$ & 24,9 & 283,58 \\
\hline
\end{tabular}

Tabla 5. Resultados de viscosidad del equipo N3 obtenidos con el viscosímetro de caída de bolas.

\begin{tabular}{cccccc}
\hline $\begin{array}{c}\text { Ensa } \\
\text { yo }\end{array}$ & $\begin{array}{c}\text { Temperat } \\
\text { ura } \\
{\left[{ }^{\circ} \mathbf{C}\right]}\end{array}$ & $\begin{array}{c}\text { Distan } \\
\text { cia } \\
{[\mathbf{m m}]}\end{array}$ & $\begin{array}{c}\text { Tiem } \\
\text { po } \\
{[\mathbf{s}]}\end{array}$ & $\begin{array}{c}\text { Visc. } \\
\text { Dinám } \\
\text { ica } \\
{[\text { Pa.s] }}\end{array}$ & $\begin{array}{l}\text { Visc. } \\
\text { Cinemát } \\
\text { ica } \\
{[\mathbf{c S t}]}\end{array}$ \\
\hline $\begin{array}{c}\text { Limp } \\
\text { io }\end{array}$ & 23 & 18,2 & 0,45 & 2,39 & 262,35 \\
$\mathbf{1}$ & 23 & 18,2 & 0,41 & 2,49 & 283,58 \\
$\mathbf{2}$ & 23 & 18,2 & 0,41 & 2,49 & 283,58 \\
$\mathbf{3}$ & 23 & 18,2 & 0,41 & 2,49 & 283,58 \\
\hline
\end{tabular}

Como se observa en la Tabla 6, en el equipo N4 la viscosidad cinemática disminuye un $4,6 \%$, pérdida que se mantiene de cambio a cambio.

Tabla 6. Resultados de viscosidad del equipo N4 obtenidos con el viscosímetro de caída de bolas

\begin{tabular}{cccccc}
\hline $\begin{array}{c}\text { Ensa } \\
\text { yo }\end{array}$ & $\begin{array}{c}\text { Temperat } \\
\text { ura } \\
{\left[{ }^{\circ} \mathbf{C}\right]}\end{array}$ & $\begin{array}{c}\text { Distan } \\
\text { cia } \\
{[\mathbf{m m}]}\end{array}$ & $\begin{array}{c}\text { Tiem } \\
\text { po } \\
{[\mathbf{s}]}\end{array}$ & $\begin{array}{c}\text { Visc. } \\
\text { Dinám } \\
\text { ica } \\
{[\text { Pa.s] }}\end{array}$ & $\begin{array}{l}\text { Visc. } \\
\text { Cinemát } \\
\text { ica } \\
{[\mathbf{c S t}]}\end{array}$ \\
\hline $\begin{array}{c}\text { Limp } \\
\text { io }\end{array}$ & 21,5 & 18,2 & 0,41 & 24,3 & 290,10 \\
$\mathbf{1}$ & 21,5 & 18,2 & 0,41 & 24,9 & 276,76 \\
$\mathbf{2}$ & 21,5 & 18,2 & 0,41 & 24,9 & 276,76 \\
$\mathbf{3}$ & 21,5 & 18,2 & 0,41 & 24,9 & 276,76 \\
\hline
\end{tabular}

Como se puede apreciar en la Tabla 7, en el equipo N5 la viscosidad cinemática disminuye un 6,7 \% y esta pérdida se mantiene de cambio a cambio.

\section{Tabla 7.Resultados de viscosidad del equipo N5} obtenidos con el viscosímetro de caída de bolas

\begin{tabular}{cccccc}
\hline $\begin{array}{c}\text { Ensa } \\
\text { yo }\end{array}$ & $\begin{array}{c}\text { Temperat } \\
\text { ura } \\
{\left[{ }^{\circ} \mathbf{C}\right]}\end{array}$ & $\begin{array}{c}\text { Distan } \\
\text { cia } \\
{[\mathbf{m m}]}\end{array}$ & $\begin{array}{c}\text { Tiem } \\
\text { po } \\
{[\mathbf{s}]}\end{array}$ & $\begin{array}{c}\text { Visc. } \\
\text { Dinám } \\
\text { ica } \\
{[\text { Pa.s] }}\end{array}$ & $\begin{array}{l}\text { Visc. } \\
\text { Cinemát } \\
\text { ica } \\
{[\mathbf{c S t}]}\end{array}$ \\
\hline $\begin{array}{c}\text { Limp } \\
\text { io }\end{array}$ & 23 & 12 & 0,60 & 56,25 & 639,17 \\
$\mathbf{1}$ & 23 & 12 & 0,59 & 55,30 & 596,35 \\
$\mathbf{2}$ & 23 & 12 & 0,59 & 55,30 & 596,35 \\
$\mathbf{3}$ & 23 & 12 & 0,59 & 55,30 & 596,35 \\
\hline
\end{tabular}

\section{DISCUSIÓN DE RESULTADOS}

En esta sección se analizan por separado los resultados de las ensayos realizados empleando el EPR en desarrollo.

\subsection{De la mancha de aceite}

En las Figuras 9(a) y 9(b) se comparan los resultados obtenidos para el indicé de dispersión tanto de motores diesel como de gasolina.

Como se puede apreciar en la Figura 9(a) para los vehículos con motor diesel en las muestras correspondientes a los cambios de aceite el índice de dispersión se mantuvo entre $74 \%$ y $78 \%$. En consecuencia se puede tomar el promedio de estos valores como nivel de alerta para el cambio oportuno del lubricante para las flotas de automovilesen servicio en las mismas condiciones de trabajo. 

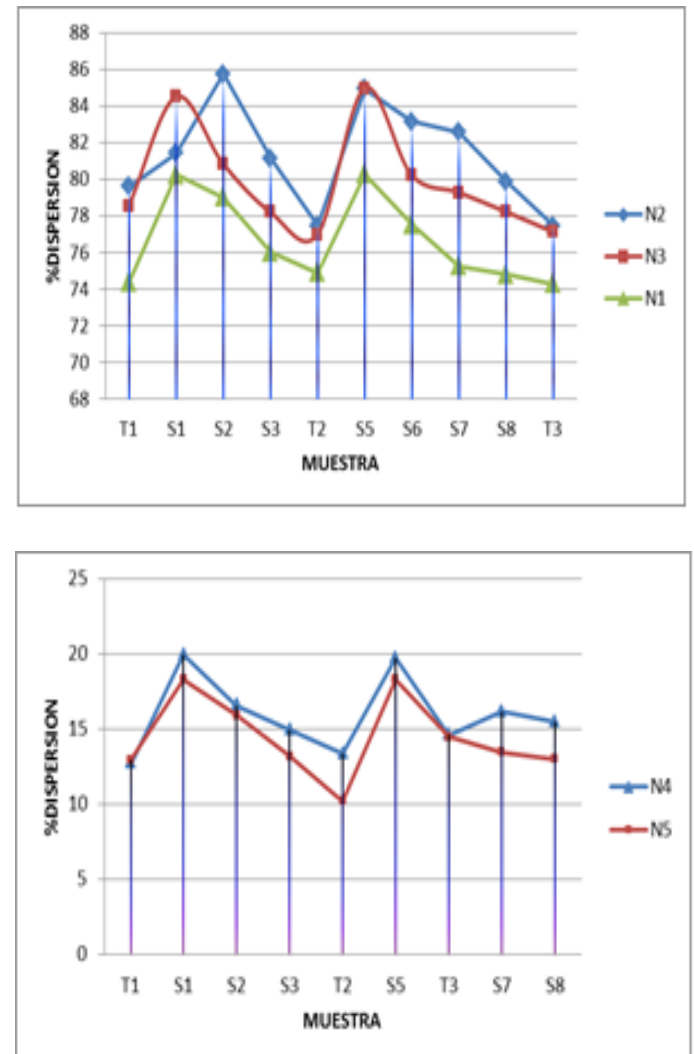

Figura 9. (a) Índice de dispersión en motores diésel, (b) índice de dispersión en motores de gasolina.

Con respecto a la Figura 9(b) se puede apreciar que la diferencia de marcas, modelos y lugar de trabajo no intervienen en la dispersión, ya que esta se mantiene en un rango de 10 a $15 \%$.En consecuencia se puede tomar el promedio de estos valores como nivel de alerta, para el cambio oportuno del lubricante en las mismas condiciones de trabajo.

\subsection{Crepitación}

Las pruebas realizadas con esta técnica se comparan en la Figura 4 que muestra la aparición de pequeñas burbujas de agua que no crepitan. En cuanto a los equipos diesel dicho contenido es evidente después de la tercera semana, cuando aparecen burbujas de 0.2 a $0.5 \mathrm{~mm}$ lo que indica que el contenido de agua: de 500 a 1000 ppm se produce por la condensación de humedad del ambiente de trabajo. Con respecto a los motores de gasolina es notable la presencia de burbujas con un diámetro máximo de $0.5 \mathrm{~mm}$, desde la primera semana de realizado el cambio. Este tamaño indica que estos equipos contienen entre $0.05-0.1 \%$ de agua originada por la condensación debida al cambio de clima, ya que éstos trabajan en rutas intermunicipales.

\subsection{Contenido de partículas ferromagnéticas}

Los resultados de la detección de partículas ferromagnéticas permiten determinar su presencia, forma y tamaño en el lubricante de motores diesel pero no permiten evaluar de manera visible un aumento o disminución del número de partículas en la muestra, por lo tanto, se considera que esta prueba produce como resultado: contiene o no contiene partículas ferromagnéticas.

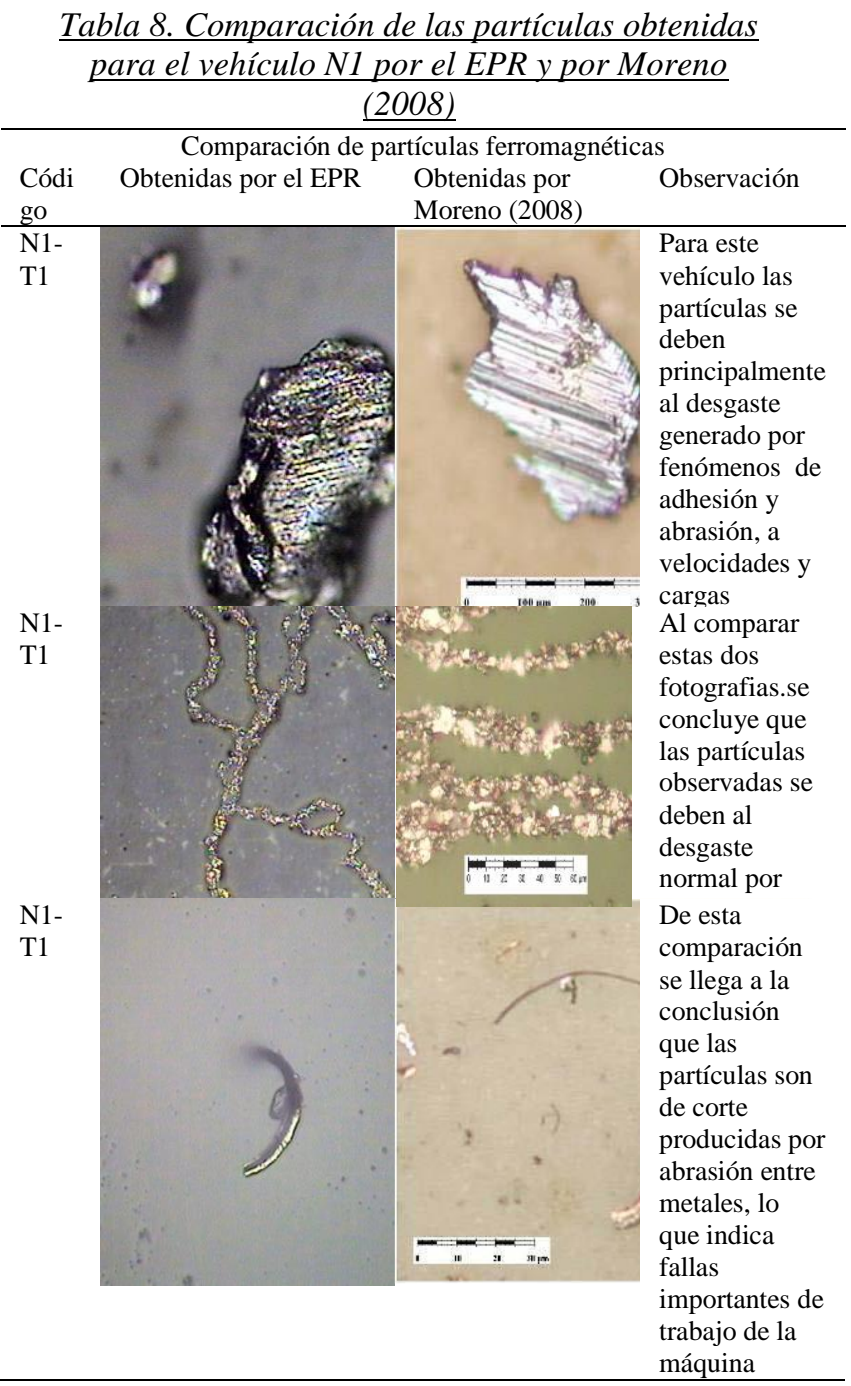

A partir de las muestras obtenidas con el microscopio Bosh se realiza un estudio comparado de la forma de las partículas presentes en las muestras tomadas con el EPR y las obtenidas por Moreno (2008) con aumentos semejantes. En la 
Tabla 8 se muestra dicha comparación para el vehículo N1.

El equipo N2 presenta partículas similares a las del equipo $\mathrm{N} 1$ y en cantidad semejante de lo que se concluye, que su desgaste es producido por los mismos mecanismos. En relación con el equipo N3 se observa que presenta partículas similares a las del equipo $\mathrm{N} 1$ pero en menor cantidad de lo que se deduce que el equipo tiene un desgaste normal y está en mejores condiciones en comparación de los otros equipos diesel. Respecto a los equipos N4 y N5 de gasolina, no se observan partículas de desgaste debido a que estos motores son limpios, están en muy buenas condiciones y realizan su cambio de lubricante en el kilometraje recomendado por el fabricante.

\subsection{Viscosímetro de caída de bolas}

Los equipos con motor diesel presentan un aumento promedio de la viscosidad de $8.5 \%$. Este aumento, no sobrepasa el nivel de alarma que corresponde al $40 \%$, lo que indica que no hay contaminación alarmante del lubricante que debe estar cumpliendo sus funciones detergentes/ dispersantes, recogiendo los residuos generados por la combustión.

Los equipos con motor de gasolina presentan una disminución promedio de la viscosidad de $6 \%$, que no sobrepasa el nivel de alarma que corresponde al $15 \%$, lo que indica que no hay dilución por combustible o que se haya añadido aceite con menor viscosidad.

\section{CONCLUSIONES}

La prueba de la mancha de aceite se puede caracterizar con el porcentaje de dispersión que de acuerdo con los resultados obtenidos puede servir como criterio para determinar el periodo de cambio de aceite.

La prueba de crepitación se constituye en un ensayo sencillo y con una escala de cuantificación de la humedad de fácil observación.

La viscosidad del lubricante de los equipos diesel aumenta con el uso mientras que la de los equipos de gasolina disminuye con el uso.

Si en el ensayo de partículas ferromagnéticas se emplea un microscopio que no hace parte del equipo, se obtiene información detallada del tipo de desgaste presente.

\section{REFERENCIAS}

Fygueroa S. (2005). "Técnicas de seguimiento del estado de motores diesel". Revista colombiana de tecnologías de avanzadas. Vol. 1, No. 5v

Fygueroa S., S. J., Serrano R., J. C. y Moreno C., G. G. (2009). "Mantenimiento predictivo de motores mediante análisis de aceite". Revista colombiana de tecnologías de avanzadas. Vol. 1, No. 13

Fygueroa S., S. J., Serrano R., J. C. y Moreno C., G. G. (2009). "A method to evaluate metallic contents in used oil smples". Revista colombiana de tecnologías de avanzadas. Vol. 2, No. 8

Delgado S., J. C., Fygueroa S., S, J. y Sierra, S. E. (2016). "Diseño de un equipo de pruebas rápidas para aceites usados". INGE CUC. Vol. 12, no. 1 ,

Bilbao, M. (2005). Ensayo de la mancha, Wearcheck ibérica. http://clientes.lubricationmanagement.com/Documentacion/DocTecnic a/Mancha.asp, (Consultado: 23 mayo 2017)

Sam, G., Santhosh, B., Vishaal, G. y Mridul, G. (2006) "Effect of soot on lubricant oil viscosity". Tribology International. No. 40.

Moreno, L. (2008). "Contribución al desarrollo y mejora de técnicas para la detección y análisis de partículas metálicas contaminantes en aceites lubricantes usados". Tesis de doctorado. Universidad Politécnica de Valencia. Valencia, España.

Widman, R. (2005). "La contaminación del aceite por agua y su detección en campo". Widman International SRL. http://docplayer.es/4600575-Lacontaminacion-del-aceite-por-agua-y-sudeteccion-en-campo-por-richardwidman.html, (Consultado 6 de octubre de 2017)

Abellaneda, G. y Pigeon, D. (2011). "Lubricating Oils Evaluation of Dispersancy Capacity of Lubricating Oils and the Impact of Biofuels on Lubricant Dispersancy". Journal of ASTM International, Vol. 8, No. 7. 